\title{
To Study the Combining Ability and Gene Action Involve in Expression of Traits in Pumpkin (Cucurbita moschata Duch. ex. Poir)
}

\author{
M.K. Singh ${ }^{1 *}$, V.B. Singh ${ }^{1}$, G.C. Yadav ${ }^{1}$ and Pushpendra Kumar ${ }^{2}$ \\ ${ }^{I}$ Department of Vegetable Science, NDUA \&T, Ayodhya (U.P.), India \\ ${ }^{2}$ Faculty of Agriculture, Bindeshwari P. G. College Akbarpur, Ambedkar Nagar, (U.P.), India \\ *Corresponding author
}

\section{Keywords}

Pumpkin

(Cucurbita

moschata Duch. ex.

Poir), Combining

ability and gene

action

Article Info

Accepted:

12 May 2019

Available Online:

10 June 2019

\section{A B S T R A C T}

The present investigation was carried out with aims to assess the combining ability involving 7 parents namely, Narendra Agrim $\left(\mathrm{P}_{1}\right)$, Narendra Amrit $\left(\mathrm{P}_{2}\right)$, Narendra Upkar $\left(\mathrm{P}_{3}\right)$, NDPK-76-1 $\left(\mathrm{P}_{4}\right)$, NDPK-724 $\left(\mathrm{P}_{5}\right)$ and NDPK-39-2 $\left(\mathrm{P}_{6}\right)$ and NDPK-12-10 $\left(\mathrm{P}_{7}\right)$ of pumpkin and their $21 \mathrm{~F}_{1}$ hybrids produced in diallel mating design excluding reciprocals at MES, Vegetable Science, NDUA.\&T, Kumarganj, Faizabad (U.P.) India in Zaid, 2016. The experiments were laid out in RBD with three replications having each experimental unit of single row with spacing of $3.0 \mathrm{~m}$ $(\mathrm{R} \times \mathrm{R}) \times 0.5 \mathrm{~m}(\mathrm{P} \times \mathrm{P})$. The observations were recorded on parents and $\mathrm{F}_{1}$ 's for twenty quantitative traits including six quality traits $v i z$., node number to first male flower appearance, node number to first female flower appearance, days to first male flower anthesis, days to first female flower anthesis, days to first fruit harvest, vine length $(\mathrm{m})$, internodal length $(\mathrm{cm})$, number of primary branches per plant, fruit weight $(\mathrm{kg})$, number of fruits per plant, equatorial circumference of fruit $(\mathrm{cm})$, polar circumference of fruit $(\mathrm{cm})$, flesh thickness $(\mathrm{cm})$, fruit yield per plant $(\mathrm{kg})$, and six quality/biochemical traits namely ascorbic acid, reducing sugars (\%) non-reducing sugars $(\%)$, total sugars $(\%)$, dry matter content and total soluble solids. The analysis of variance for combining ability revealed that there were significant differences for all the traits. Mean squares due to general combining ability were observed significant for all the traits and specific combining ability were observed significant for all traits except days first fruit harvest and average fruit weight. The parents Narendra Upkar $\left(\mathrm{P}_{3}\right)$, NDPK-39-2 $\left(\mathrm{P}_{6}\right)$ and NDPK-12-10 $\left(\mathrm{P}_{7}\right)$ were found good general combiner for fruit yield per plant and number of fruits per plant. Out of the best five significant specific crosses with high per se performance for fruit yield per plant, three crosses NDPK-76-1 x NDPK-39-2 $\left(\mathrm{P}_{4} \times \mathrm{P}_{6}\right)$, Narendra Upkar $\mathrm{x}$ NDPK-12-10 $\left(\mathrm{P}_{3} \times \mathrm{P}_{7}\right.$ ) and (Narendra Upkar x NDPK-724) $\mathrm{P}_{3} \times \mathrm{P}_{5}$ indicated good specific combing ability. Both additive and dominance variance were found important in the inheritance of most of the traits, whereas dominance variance were found more prominent than the additive variance. Average degree of dominance revealed the presence of over dominance for all the traits. The proportions of genes $\left(\hat{\mathrm{H}}_{2} / 4 \hat{\mathrm{H}}_{1}\right)$ in the parents were less than 0.25 for all the traits indicating asymmetrical distribution of alleles at loci showing dominance. In general the proportions of dominant and recessive genes in parents $\left.\left[4 \hat{\mathrm{D}} \hat{\mathrm{H}}_{1}\right)^{1 / 2}+\hat{\mathrm{F}} /\left(4 \hat{\mathrm{D}} \hat{\mathrm{H}}_{1}\right)^{1 / 2}-\hat{\mathrm{F}}\right]$ were more than unity with positive $\hat{\mathrm{F}}$ value for all traits indicating major contribution of dominant genes. 


\section{Introduction}

Pumpkin (Cucurbita moschata Duch. ex. Poir) is one of the most important vegetable crop of family Cucurbitaceae. It is grown throughout the world due to its good nutritional value and also higher returns to the farmers. The centre of orgin of pumpkin is central Mexico. Pumpkin is a herbaceous annual, sexually propagated vegetable allopolyploid having chromosome number $2 n=2 x=40$. Stem is angular, five ridged without hairs, trailing and branched. Trailing vine strikes roots at nods. Leaves are deeply or shallowly lobed not pinnatifid. Fruits have diuretic and vermetic action. Seeds are non bitter, tasty and nutritious. The flowers are large and yellow coloured with showy companulate corolla. Ovary is inferior and tricarpellary. Corolla is companulate, gamopetalous, lobed. Plants are monoecious, highly cross pollinated, entamophilous with three anthers. The word pumpkin was derived from the Greek word pepon, which means "large melon", something round and large.

Based on commercial significance the cultivated Cucurbita species rank among the 10 leading vegetable crops worldwide. China and India lead the world production and other major producers are U.S., Egypt, Mexico, Ukraine, Cuba, Italy, Iran and Turkey (Ferriol and Pico, 2008). The total area of pumpkin in India is 19,760 hectares whereas, the total production is 0.42 million tonne with productivity $21.25 \mathrm{mt} / \mathrm{ha}$ (Annonymous, 2015).

Combining ability analysis helps in the evaluation of inbreds in terms of genetic value and in the selection of suitable parent for hybridization. The superior specific cross combinations are also identified by this technique. The information regarding nature of gene action controlling important quantitative traits is required for devising a suitable breeding strategy and for improvement of pumpkin.

The existence of significant amount of nonadditive gene action is a pre requisite for exploitation of heterosis. The specific combining variance largely is the measure of dominance variance. If heterosis is high for specific cross and observations made are true for economic trait like yield, it is possible to utilize the cross (Arunachalam, 1989).

The three economically important species, $C$. pepo, C. moschata, and C. maxima are highly polymorphic in fruit characteristics, inspiring much research into their inheritance although most of such studies have been done in $C$. pepo and $C$. maxima. The gene list of Cucurbita sp. includes 79 loci for phenotypic/morphological traits and 48 polymorphic allozyme loci along with linkage and gene mapping (Paris and Brown, 2005). However, information on simply inherited characters documented in the gene list is almost lacking for C. moschata, and among the few that have been reported so far are Trifluralin (a herbicide) tolerance controlled by a dominant gene " $T$ " (Adeoye and Coyne, 1981), naked kernel (absence of tough seed coat) trait controlled by a pair of recessive genes (Zhou et al., 1995) and mottled light and dark green fruit colour, monogenic dominant ("Mldg") over dark green fruit (Cardoso et al., 1995). A wide range of expression of the trait "silver leaf mottling" was observed in C. moschata although all the reports indicated that this trait showed dominance over non-mottled leaf. Ribeiro and da Costa (1989) reported that a partially dominant gene was responsible for silver mottling in leaf but modifier genes affected its expression while it was reported to be conditioned by two genes (Latha and Gopalkrishnan, 1993) and a single gene " $M$ " (Wessel-Beaver and Katzir, 2000). Information on polygenic inheritance of 
quantitative characters in pumpkin is also meager. Non-additive gene action for vine length, number of laterals, nodes of first staminate and pistillate flowers and days to first staminate and pistillate flowers (Doijode and Sulladmath, 1988); for number of seeds/fruit, 100-seed weight and seed size index (Doijode et al., 1987); over-dominance (Galka, 1987) and dominance (Sirohi et al., 1986) for fruit weight; over-dominance for vine length, fruit number/plant, flesh thickness, fruit shape index and fruit yield per plant (Sirohi et al., 1986) and over-dominance gene action for all the nutritional traits, namely, total soluble solids, carotenoids, ascorbic acid, calcium, and iron content (Sirohi and Yayasani, 2001) clearly suggested the importance of exploiting heterosis commercially.

\section{Materials and Methods}

The experimental materials for the present study comprised of six promising and diverse inbreds and varieties of pumpkin selected on the basis of genetic variability from the germplasm stock maintained in the Department of Vegetable Science, N.D. University of Agriculture \& Technology, Kumarganj, Faizabad (U.P.) India. The selected parental lines i.e. Narendra Agrim $\left(\mathrm{P}_{1}\right)$, Narendra Amrit $\left(\mathrm{P}_{2}\right)$, Narendra Upkar $\left(\mathrm{P}_{3}\right)$, NDPK-76-1 ( $\left.\mathrm{P}_{4}\right), \quad$ NDPK-724 $\left(\mathrm{P}_{5}\right)$, NDPK-39-2 $\left(\mathrm{P}_{6}\right)$ and NDPK-12-10 $\left(\mathrm{P}_{7}\right)$ were raised and crossed in the all possible combinations, excluding reciprocals, during summer, 2016 to develop $21 \mathrm{~F}_{1}$ hybrids. The experiments were conducted in Randomized Block Design (RBD) with three replications to assess the performance of $21 \mathrm{~F}_{1}$ hybrids and 7 parents. The treatments were planted in row to row at $3.0 \mathrm{~m}$ apart with a plant to plant spacing of $0.50 \mathrm{~m}$. The seeds were sown on $9^{\text {th }}$ March, 2017. All the recommended agronomic package of practices and plant protection measures were followed to raise good crop. Observations were recorded on fourteen economic traits including biochemical analysis viz., node number to first male flower, node number to first female flower, days to first male flower anthesis, days to first female flower anthesis, days to first marketable fruit harvest, number of primary branches per plant, equatorial circumference of fruit $(\mathrm{cm})$, polar circumference of fruit $(\mathrm{cm})$, flesh thickness $(\mathrm{cm})$, internodal length $(\mathrm{cm})$, vine length $(\mathrm{m})$, average fruit weight $(\mathrm{kg})$, number of fruits per plant, fruit yield per plant $(\mathrm{kg})$, ascorbic acid $(\mathrm{mg} / 100 \mathrm{~g})$, reducing sugars $(\%)$, nonreducing sugars $(\%)$, total sugars $(\%)$, dry matter content $(\%)$ and total soluble solids $(\%)$.The analysis of variance was carried out as suggested by Panse and Sukhatme (1967).

\section{Results and Discussion}

Combining ability analysis is considered to be an effective tool for classifying parental lines in terms of their hybrid performance and better understanding of the nature of quantitatively inherited traits. $\mathrm{Per}$ se performance has been important criteria for selection of parents for making crosses by the breeder but good performing parental lines do not always produce desirable $F_{1}$. The information regarding combining ability of the parents, for yield and various yield attributes becomes relevant to the plant breeders for chalking out an efficient breeding methodology. Among the several techniques available for quantitative genetic analysis, diallel cross analysis has usually been found to be efficient and easiest method to identify the best donors for hybridization.

In pumpkin, combining ability analysis have been made for two seasons in $F_{1}$ crosses following diallel analysis by Pandey et al., 2010; Nisha and Veeraragavathatham, 2014. The important findings of combining ability obtained using diallel analysis consisting 
seven parental lines and $21 \mathrm{~F}_{1}$ hybrids have been discussed as follows:

The analysis of variance (Table 1) for combining ability revealed that there were significant differences for all the traits. Mean squares due to general combining ability were observed significant for all the traits whereas specific combining ability were observed significant for all the traits except days to first fruit harvest and average fruit weight.

The estimates of highly significant gca and sca variances revealed that both additive and non-additive gene action were important in the expression of all twenty traits studied. Thus, both selection and heterosis breeding methods respectively might be advantageous for effective utilization of additive and nonadditive genetic variances for improvement of these traits. Similar findings had also been reported by Singh et al., (2005), Dubey and Maurya (2006) and Rehana and Sharma (2007) in bottle gourd. Variation in the magnitude of gca and sca variances were also observed which may be due to environmental influence. Similar findings were also reported by Maurya (1994) in pumpkin.

General combining ability study helps in making the choice of the parents and also helps in the isolation of suitable germplasm for further improvement. General combining ability is primarily a function of additive and additive xadditive gene action.

Parent, $\mathrm{P}_{1}$ (Narendra Agrim) had good general combining ability for days to first male flower anthesis, days to first female flower anthesis, node number to first male flower appearance, node number to first female flower appearance, number of primary branches per plant, dry matter content, total sugars reducing sugar and non-reducing sugars. The parent $\mathrm{P}_{2}$ (Narendra Amrit) had good GCA effects for equatorial circumference of fruit, polar circumference, flesh thickness and Internodal length. The parent $\mathrm{P}_{3}$ (Narendra Upkar) showed good general combining ability effects for days to first fruit harvest, vine length, average fruit weight and fruit yield per plant. The parent $\mathrm{P}_{4}$ (NDPK-76-1) had good general combining ability for node number to first male flower appearance, node number to first female flower appearance, reducing sugars and total sugars. The parent, $\mathrm{P}_{5}$ (NDPK-724) had good general combining ability for ascorbic acid content, days to first female flower anthesis, node number to first male flower appearance, equatorial circumference of fruit, dry matter content and reducing sugar. The parent, $\mathrm{P}_{6}$ (NDPK-39-2) was found good general combiner for internodal length, vine length, number of fruits per plant, fruit yield per plant and total soluble solids. $\mathrm{P}_{7}$ (NDPK-12-10) had good GCA effects for days to first fruit harvest, vine length, number of fruits per plant and fruit yield per plant. Good general combining ability for various traits has also been reported by earlier researchers viz., fruit yield per plant (Kathiria et al., 2005; Singh et al., 2005 in bottle gourd and Pandey et al., 2010; Nisha and Veeraragavathatham, 2014 in pumpkin) fruit weight (Nisha and Veeraragavathatham, 2014) vine length (Nisha and Veeraragavathatham, 2014) flesh thickness (Pandey et al., 2010; Nisha and Veeraragavathatham, 2014) total carotenoids (Pandey et al., 2010) ascorbic acid (Pandey et al., 2010) earliness (Nisha and Veeraragavathatham, 2014) which corroborate the present findings.

The ranking of desirable parents on the basis of GCA effects for twenty traits revealed that it was difficult to pickup a single good general combiner for all traits. However, parents $\mathrm{P}_{6}$ (NDPK-39-2) and $\mathrm{P}_{3}$ (Narendra upkar) were found as good general combiner for number of fruits per plant and fruit yield per plant. Parents $\mathrm{P}_{1}$ (Narendra Agrim) and $\mathrm{P}_{2}$ 
(Narendra Amrit) also emerged as good general combiner for maturity traits (earliness), number of primary branches, internodal length, whereas parents $\mathrm{P}_{1}$ (Narendra Agrim) and $\mathrm{P}_{3}$ (Narendra upkar) were found good as well as average general combiner for biochemical traits (dry matter, total sugars, reducing and non-reducing sugars and total soluble solids). This showed that parents having good gca effects for yield also had good gca effects for one or more yield components.

Further, the inbred mentioned above may serve as valuable donors for hybridization programme and may be choosen as most preferred parents for breeding of early maturing high yielding hybrids with good quality traits in pumpkin. In general, the parents which gave best per se performance were also the best general combiners indicating a positive association between the two parameters. Similar correspondence between these two parameters was also observed by Maurya (1994) and Kumar (2000) in case of bottle gourd. The parents both for gca effect and per se performance suggested that mean value of parents for a particular traits may have some predictive significance of its gca effect for that trait (Table 2).

Thus, parents $\mathrm{P}_{1}$ (Narendra Agrim), $\mathrm{P}_{5}$ (NDPK-724) and $\mathrm{P}_{6}$ (NDPK-39-2) emerged as the most useful parent as either one or both of them were found good general combiner along with high per se performance for most of the traits studied. Hence, these three parents may also be recommended for exploitation in hybridization programme aimed at improving the yield components for which they were good general combiner (Table 2).

The SCA effects represent non-additive gene action which is non-fixable. Specific combining ability effects helps in the identification of superior cross combinations for development of promising varieties/hybrids. The crosses showing high SCA effects involving parents with high GCA effects may give rise to desirable segregants in future generation. The specific combining ability effects of the twenty one crosses for twenty traits have been presented in Table-3. Perusal of Table-3 revealed that significant positive and negative sca effects were observed for all the characters. However, none of the crosses had significant sca effects for all the traits. Maurya (1994) also reported similar results. The specific combining ability effects of the crosses showing significant sca effects for fruit yield and their relationship with other yield components had been summarized in Table-3. Six crosses had significant sca effects in desirable direction for fruit yield per plant. These crosses were found to be significant specific combiners for one or more yield components as well. Development of high yielding $F_{1}$ coupled with more number of fruits per plant is an important aspect. Out of twenty one crosses, six crosses which showed significant sca effects for fruit yield per plant namely, $\mathrm{P}_{1} \times$ $\mathrm{P}_{7}, \mathrm{P}_{2} \times \mathrm{P}_{7}, \mathrm{P}_{3} \times \mathrm{P}_{5}, \mathrm{P}_{3} \times \mathrm{P}_{7}, \mathrm{P}_{4} \times \mathrm{P}_{5}$ and $\mathrm{P}_{4} \times$ $\mathrm{P}_{6}$ which exhibited significant sca effects both for fruit yield. Therefore, these crosses may likely to fit for farmers demand and also throw good transgressive segregates combining high fruit yield in later generations of selection. There was a close correspondence between sca effect.

Perusal of data presentation in Table 3 revealed that six cross combinations showed significant and positive specific combining ability effect for fruit yield involving parents with Low $x$ High $(\mathrm{L} \times \mathrm{H})$, Low $\times$ Low $(\mathrm{L} \times$ L), High $\times$ Low $(\mathrm{H} \times \mathrm{L})$ and High $\times$ High $(\mathrm{H} \times$ H) general combining ability effects for fruit yield. Similar results have also been reported by Maurya (2010). 
Table.1 Analysis of variance (mean squares) for combining ability in 7 x 7 diallel crosses of pumpkin

\begin{tabular}{|c|c|c|c|c|c|c|c|c|c|c|c|}
\hline $\begin{array}{c}\text { Source } \\
\text { of } \\
\text { Variatio } \\
\text { n }\end{array}$ & d.f. & 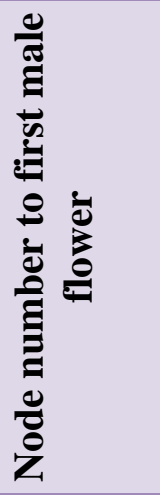 & 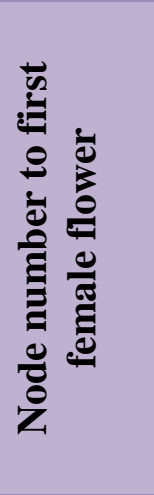 & 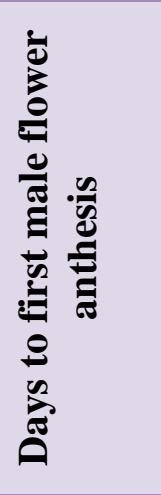 & 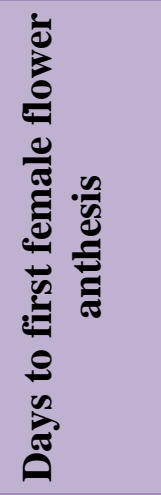 & 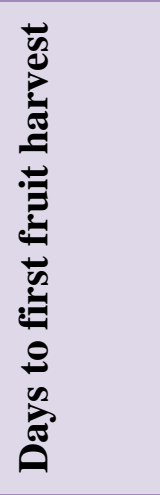 & 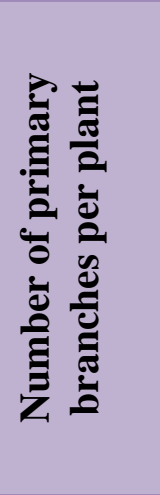 & 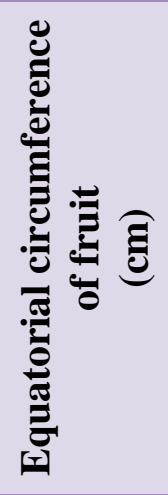 & 泀 & 造 & 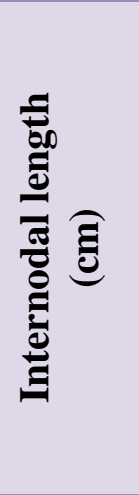 \\
\hline GCA & 6 & $9.74 * *$ & $18.51 * *$ & $75.89 * *$ & $33.03 * *$ & $25.20 * *$ & $1.74 * *$ & $77.95 * *$ & $50.93 * *$ & $0.49 * *$ & $1.99 * *$ \\
\hline SCA & 21 & $0.64 * *$ & $6.62 * *$ & $10.92 * *$ & $5.25 *$ & 8.55 & $0.86^{* *}$ & $11.87 * *$ & $12.03 * *$ & $0.08 * *$ & $0.46^{* *}$ \\
\hline Error & 54 & 0.03 & 0.88 & 3.36 & 2.80 & 5.39 & 0.10 & 4.49 & 3.69 & 0.01 & 6.28 \\
\hline
\end{tabular}

Table.1 Contd....

\begin{tabular}{|c|c|c|c|c|c|c|c|c|c|c|c|}
\hline $\begin{array}{l}\text { Source of } \\
\text { Variation }\end{array}$ & d.f. & 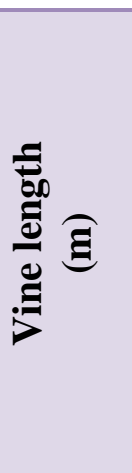 & 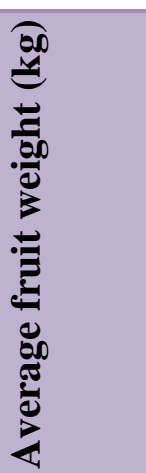 & 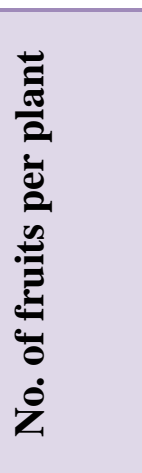 & 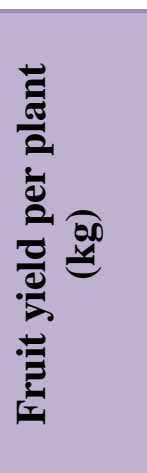 & 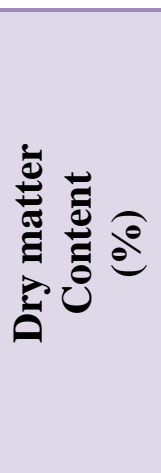 & 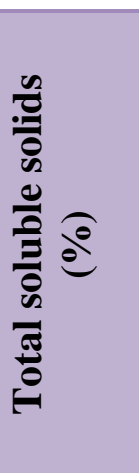 & 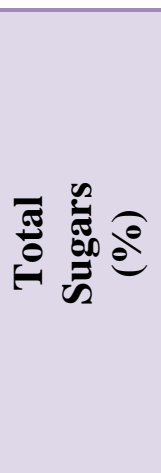 & 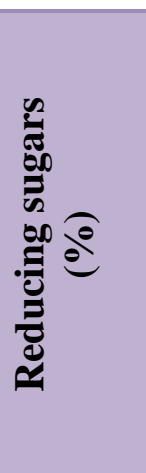 & 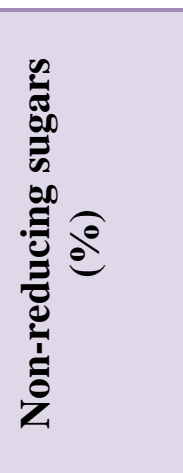 & 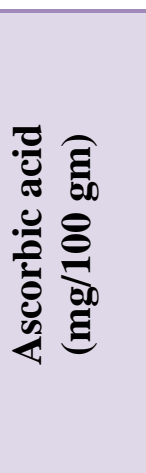 \\
\hline GCA & 6 & $1.32 * *$ & $0.07 * *$ & $0.63 * *$ & $2.74 * *$ & $1.21 * *$ & $0.20^{* *}$ & $0.51 * *$ & $0.23 * *$ & $0.24 * *$ & $0.18 * *$ \\
\hline SCA & 21 & $0.72 * *$ & 0.01 & $0.11 * *$ & $1.50 * *$ & $0.50 * *$ & $0.55^{* *}$ & $0.32 * *$ & $0.20 * *$ & $0.14 * *$ & $1.30 * *$ \\
\hline Error & 54 & 0.02 & 0.01 & 0.02 & 0.12 & 0.03 & 0.02 & 0.03 & 0.01 & 0.00 & 0.03 \\
\hline
\end{tabular}

*,** Significant at 5 per cent and 1 per cent probability levels, respectively. 
Table.2 Estimates of GCA effects of parents in 7 x 7 diallel cross of pumpkin

\begin{tabular}{|c|c|c|c|c|c|c|c|c|c|c|}
\hline Traits & $\begin{array}{c}\text { Node } \\
\text { number } \\
\text { to first } \\
\text { male } \\
\text { flower }\end{array}$ & $\begin{array}{c}\text { Node } \\
\text { number } \\
\text { to first } \\
\text { female } \\
\text { flower }\end{array}$ & $\begin{array}{c}\text { Days to } \\
\text { first } \\
\text { male } \\
\text { flower } \\
\text { anthesis }\end{array}$ & $\begin{array}{l}\text { Days to } \\
\text { first } \\
\text { female } \\
\text { flower } \\
\text { anthesis }\end{array}$ & $\begin{array}{c}\text { Days to } \\
\text { first } \\
\text { marketable } \\
\text { fruit } \\
\text { harvest }\end{array}$ & $\begin{array}{c}\text { Number } \\
\text { of } \\
\text { primary } \\
\text { branches } \\
\text { per } \\
\text { plant }\end{array}$ & $\begin{array}{l}\text { Equotorial } \\
\text { circumference } \\
\text { of fruit }(\mathbf{c m})\end{array}$ & $\begin{array}{c}\text { Polar } \\
\text { circumference } \\
\text { of fruit }(\mathbf{c m})\end{array}$ & $\begin{array}{c}\text { Flesh } \\
\text { thickness } \\
(\mathrm{cm})\end{array}$ & $\begin{array}{l}\text { Internodal } \\
\text { length } \\
(\mathrm{cm})\end{array}$ \\
\hline $\mathbf{P}_{1}$ & $-0.17 * *$ & $-1.45^{* *}$ & $-3.11 * *$ & $-2.05 * *$ & -0.44 & $0.43 * *$ & $-3.10 * *$ & 0.49 & $-0.22 * *$ & 0.13 \\
\hline $\mathbf{P}_{2}$ & $-0.41 * *$ & $-1.42 * *$ & $3.10 * *$ & $2.69 * *$ & $2.34 * *$ & $-0.21 *$ & $2.48 * *$ & $1.52 *$ & $0.50 * *$ & $-0.45 * *$ \\
\hline $\mathbf{P}_{3}$ & $0.42 * *$ & $1.84 * *$ & $-2.80 * *$ & $-1.68 * *$ & $-2.57 * *$ & $-0.52 * *$ & $2.09 * *$ & $1.72 * *$ & -0.04 & $-0.49 * *$ \\
\hline $\mathbf{P}_{4}$ & $-0.88 * *$ & $-1.21 * *$ & 0.28 & 0.04 & 0.30 & $0.71 * *$ & $-2.19 * *$ & -0.06 & $-0.10 *$ & $0.30 * *$ \\
\hline $\mathbf{P}_{5}$ & $-1.02 * *$ & -0.31 & -0.96 & $-1.13 *$ & 1.01 & $-0.29 * *$ & $4.17 * *$ & 0.67 & -0.01 & $0.63 * *$ \\
\hline $\mathbf{P}_{6}$ & -0.03 & $1.34 * *$ & -1.12 & -0.39 & 0.95 & $-0.22 *$ & -0.37 & 0.88 & $-0.15^{* *}$ & $-0.50 * *$ \\
\hline $\mathbf{P}_{7}$ & $2.08 * *$ & $1.21 * *$ & $4.61 * *$ & $2.52 * *$ & $-1.59 *$ & 0.10 & $-3.08 * *$ & $-5.22 * *$ & 0.02 & $0.37 * *$ \\
\hline SE (gi) & 0.05 & 0.29 & 0.57 & 0.52 & 0.72 & 0.10 & 0.65 & 0.59 & 0.04 & 0.11 \\
\hline SE (gi-gj) & 0.08 & 0.44 & 0.86 & 0.79 & 1.09 & 0.15 & 1.00 & 0.91 & 0.07 & 0.16 \\
\hline
\end{tabular}

Table.2 Contd...

\begin{tabular}{|c|c|c|c|c|c|c|c|c|c|c|}
\hline Parents & $\begin{array}{l}\text { Vine } \\
\text { length } \\
\text { (m) }\end{array}$ & $\begin{array}{l}\text { Average } \\
\text { fruit } \\
\text { weight } \\
\text { (kg) }\end{array}$ & $\begin{array}{c}\text { No. of } \\
\text { fruits per } \\
\text { plant }\end{array}$ & $\begin{array}{c}\text { Fruit yield } \\
\text { per plant } \\
(\mathrm{kg})\end{array}$ & $\begin{array}{c}\text { Ascorbic } \\
\text { acid } \\
\text { (mg/100) }\end{array}$ & $\begin{array}{l}\text { Reducing } \\
\text { sugars } \\
(\%)\end{array}$ & $\begin{array}{c}\text { Non- } \\
\text { reducing } \\
\text { sugars } \\
(\%)\end{array}$ & $\begin{array}{c}\text { Total } \\
\text { Sugars } \\
(\%)\end{array}$ & $\begin{array}{c}\text { Dry } \\
\text { matter } \\
\text { Content } \\
(\%)\end{array}$ & $\begin{array}{c}\text { Total } \\
\text { soluble } \\
\text { solids }(\%)\end{array}$ \\
\hline $\mathbf{P}_{1}$ & $0.09 *$ & 0.01 & $-0.23 * *$ & $-0.54 * *$ & -0.07 & $0.12 * *$ & $0.32 * *$ & $0.45 * *$ & $0.46 * *$ & -0.06 \\
\hline $\mathbf{P}_{2}$ & $-0.35 * *$ & -0.05 & $-0.27 * *$ & $-0.58 * *$ & $-0.13^{*}$ & $-0.18 * *$ & $-0.06 * *$ & $-0.23 * *$ & $-0.23 * *$ & 0.00 \\
\hline $\mathbf{P}_{3}$ & $0.49 * *$ & $0.13 * *$ & 0.06 & $0.82 * *$ & 0.02 & $-0.17 * *$ & $0.09 * *$ & $-0.11 *$ & $-0.26 * *$ & $0.10 *$ \\
\hline $\mathbf{P}_{4}$ & $-0.35 * *$ & -0.02 & 0.09 & -0.17 & $-0.11 *$ & $0.16 * *$ & -0.02 & $0.15 * *$ & $-0.27 * *$ & $-0.10 *$ \\
\hline $\mathbf{P}_{5}$ & $-0.47 * *$ & $-0.15 * *$ & $-0.27 * *$ & $-0.37 * *$ & $0.27 * *$ & $0.19 * *$ & $-0.16 * *$ & 0.03 & $0.59 * *$ & $-0.20 * *$ \\
\hline $\mathbf{P}_{6}$ & $0.34 * *$ & 0.04 & $0.39 * *$ & $0.37 * *$ & -0.08 & 0.00 & $-0.04 *$ & -0.06 & -0.03 & $0.26 * *$ \\
\hline $\mathbf{P}_{7}$ & $0.24 * *$ & 0.03 & $0.25 * *$ & $0.47 * *$ & 0.10 & $-0.11 * *$ & $-0.11 * *$ & $-0.21 * *$ & $-0.25 * *$ & 0.00 \\
\hline SE (gi) & 0.04 & 0.03 & 0.05 & 0.10 & 0.05 & 0.03 & 0.02 & 0.05 & 0.05 & 0.05 \\
\hline SE (gi-gj) & 0.06 & 0.05 & 0.07 & 0.16 & 0.08 & 0.05 & 0.03 & 0.08 & 0.08 & 0.07 \\
\hline
\end{tabular}

$\left(\mathrm{P}_{1^{-}}\right.$Narendra Agrim, $\mathrm{P}_{2}$ - Narendra Amrit, $\mathrm{P}_{3}$ - Narendra Upkar, $\mathrm{P}_{4^{-}}$NDPK-76-1, $\mathrm{P}_{5^{-}}$NDPK-724, $\mathrm{P}_{6}-\mathrm{NDPK}^{-}$-39-2, $\mathrm{P}_{7^{-}}$NDPK-12-10) 
Table.3 Estimates of SCA effects of $\mathrm{F}_{1}$ hybrids in $7 \times 7$ diallel cross of pumpkin

\begin{tabular}{|c|c|c|c|c|c|c|c|c|c|c|}
\hline Traits & $\begin{array}{c}\text { Node } \\
\text { number } \\
\text { to first } \\
\text { male } \\
\text { flower }\end{array}$ & $\begin{array}{c}\text { Node } \\
\text { number } \\
\text { to first } \\
\text { female } \\
\text { flower }\end{array}$ & $\begin{array}{c}\text { Days to } \\
\text { first } \\
\text { male } \\
\text { flower } \\
\text { anthesis }\end{array}$ & $\begin{array}{c}\text { Days to } \\
\text { first } \\
\text { female } \\
\text { flower } \\
\text { anthesis }\end{array}$ & $\begin{array}{c}\text { Days to } \\
\text { first } \\
\text { marketable } \\
\text { fruit } \\
\text { harvest }\end{array}$ & $\begin{array}{c}\text { Number of } \\
\text { primary } \\
\text { branches } \\
\text { per plant }\end{array}$ & $\begin{array}{c}\text { Equotorial } \\
\text { circumference } \\
\text { of fruit }(\mathbf{c m})\end{array}$ & $\begin{array}{c}\text { Polar } \\
\text { circumference } \\
\text { of fruit }(\mathbf{c m})\end{array}$ & $\begin{array}{c}\text { Flesh } \\
\text { thickness } \\
(\mathrm{cm})\end{array}$ & $\begin{array}{l}\text { Internodal } \\
\text { length } \\
\text { (cm) }\end{array}$ \\
\hline$P_{1} \times P_{2}$ & $0.34 *$ & -0.82 & 2.48 & -0.40 & -0.50 & 0.39 & -2.67 & 1.40 & $0.43^{* *}$ & $1.05^{* *}$ \\
\hline $\mathbf{P}_{1} \times \mathbf{P}_{3}$ & -0.23 & $1.75^{*}$ & 1.48 & 1.94 & $-4.36^{*}$ & $1.20 * *$ & $5.59 * *$ & 0.96 & 0.04 & $-0.87 * *$ \\
\hline $\mathbf{P}_{1} \times \mathbf{P}_{4}$ & $-0.33 *$ & -0.17 & $-4.54 *$ & -0.94 & 1.73 & -0.47 & 2.24 & 0.61 & -0.01 & 0.11 \\
\hline $\mathbf{P}_{1} \times \mathbf{P}_{5}$ & $0.67 * *$ & $1.84 *$ & $-6.45 * *$ & -2.38 & 2.19 & -0.47 & 1.32 & 1.81 & 0.20 & 0.47 \\
\hline $\mathbf{P}_{1} \times \mathbf{P}_{6}$ & $-0.38 *$ & 1.31 & $-6.77 * *$ & $-4.45 * *$ & $-8.78 * *$ & $1.13 * *$ & 3.55 & $5.50 * *$ & -0.06 & -0.60 \\
\hline $\mathbf{P}_{1} \times \mathbf{P}_{7}$ & $0.75 * *$ & -1.46 & 2.28 & 1.41 & 2.99 & $0.67 *$ & -2.87 & 3.47 & 0.17 & $1.10 * *$ \\
\hline $\mathbf{P}_{2} \times \mathbf{P}_{3}$ & -0.29 & $1.79 *$ & -1.63 & 0.43 & -1.18 & 0.35 & 2.97 & 1.90 & 0.22 & 0.31 \\
\hline $\mathbf{P}_{2} \times \mathbf{P}_{4}$ & $-0.39 *$ & 1.60 & -0.05 & 0.62 & -1.28 & $0.81 * *$ & $-5.58 * *$ & 1.94 & -0.03 & -0.44 \\
\hline $\mathbf{P}_{2} \times \mathbf{P}_{5}$ & -0.31 & 0.08 & 1.37 & $3.85^{*}$ & 2.51 & 0.05 & $4.33^{*}$ & -0.78 & 0.21 & $-0.71 *$ \\
\hline $\mathbf{P}_{2} \times \mathbf{P}_{6}$ & $0.76 * *$ & -0.48 & 1.62 & 1.04 & -1.99 & 0.21 & 0.86 & 1.07 & 0.22 & 0.48 \\
\hline $\mathbf{P}_{2} \times \mathbf{P}_{7}$ & $0.99 * *$ & $-2.95 * *$ & $-3.94 *$ & -2.93 & 0.54 & 0.32 & 0.21 & 0.40 & -0.21 & -0.02 \\
\hline $\mathbf{P}_{3} \times \mathbf{P}_{4}$ & 0.04 & 0.71 & 1.22 & 0.15 & 0.36 & -0.44 & 1.98 & 2.91 & 0.01 & -0.23 \\
\hline $\mathbf{P}_{3} \times \mathbf{P}_{5}$ & $-0.49 * *$ & $-4.55 * *$ & -1.30 & -0.05 & 0.25 & 0.06 & -1.51 & -1.75 & 0.19 & $1.17 * *$ \\
\hline $\mathbf{P}_{3} \times \mathbf{P}_{6}$ & $1.03 * *$ & -0.97 & 1.42 & -0.09 & 1.91 & $1.15^{* *}$ & -1.81 & 0.04 & 0.13 & 0.53 \\
\hline $\mathbf{P}_{3} \times \mathbf{P}_{7}$ & $0.59 * *$ & $-1.78 *$ & 2.09 & 2.21 & -1.38 & $0.63^{*}$ & 3.53 & 0.14 & $0.30 *$ & -0.61 \\
\hline $\mathbf{P}_{4} \times \mathbf{P}_{5}$ & $0.55 * *$ & 0.76 & 2.78 & -3.06 & -3.02 & $1.02 * *$ & -0.19 & $4.73 *$ & -0.03 & $0.84 *$ \\
\hline $\mathbf{P}_{4} \times \mathbf{P}_{6}$ & $-0.37 *$ & $2.97 * *$ & 0.50 & -0.34 & 1.24 & 0.48 & 1.87 & -1.79 & $0.41 * *$ & -0.53 \\
\hline $\mathbf{P}_{4} \times \mathbf{P}_{7}$ & 0.33 & $-3.77 * *$ & -1.85 & 1.16 & 1.68 & 0.10 & 3.08 & -0.79 & 0.11 & -0.50 \\
\hline $\mathbf{P}_{5} \times \mathbf{P}_{6}$ & $-0.47 * *$ & $-1.79 *$ & -2.91 & 2.06 & 1.63 & 0.42 & -1.09 & -1.11 & 0.03 & -0.33 \\
\hline $\mathbf{P}_{5} \times \mathbf{P}_{7}$ & $0.66 * *$ & $6.74 * *$ & 1.79 & $-3.81 *$ & -2.03 & -0.07 & 0.46 & 1.56 & 0.03 & -0.13 \\
\hline $\mathbf{P}_{6} \times \mathbf{P}_{7}$ & $1.07 * *$ & $2.32 *$ & 2.12 & 0.95 & 0.73 & 0.26 & 3.52 & $5.27 * *$ & 0.13 & $0.73 *$ \\
\hline $\operatorname{SE}\left(\mathbf{S}_{\mathrm{ij}}\right)$ & 0.16 & 0.84 & 1.65 & 1.50 & 2.08 & 0.28 & 1.90 & 1.73 & 0.13 & 0.31 \\
\hline $\operatorname{SE}\left(\mathrm{S}_{\mathrm{ij}}-\mathrm{S}_{\mathrm{ik}}\right)$ & 0.24 & 1.25 & 2.45 & 2.23 & 3.09 & 0.41 & 2.82 & 2.56 & 0.19 & 0.45 \\
\hline
\end{tabular}

*, ** Significant at 5 per cent and 1 per cent probability levels, respectively

Table.3 Contd.... 


\begin{tabular}{|c|c|c|c|c|c|c|c|c|c|c|}
\hline Traits & $\begin{array}{c}\text { Vine } \\
\text { length } \\
\text { (m) }\end{array}$ & $\begin{array}{c}\text { Average } \\
\text { fruit } \\
\text { weight } \\
\text { (kg) }\end{array}$ & $\begin{array}{c}\text { No. of } \\
\text { fruits } \\
\text { per plant }\end{array}$ & $\begin{array}{c}\text { Fruit } \\
\text { yield per } \\
\text { plant } \\
\text { (kg) }\end{array}$ & $\begin{array}{c}\text { Ascorbic } \\
\text { acid } \\
(\mathrm{mg} / 100)\end{array}$ & $\begin{array}{c}\text { Reducing } \\
\text { sugars } \\
(\%)\end{array}$ & $\begin{array}{c}\text { Non- } \\
\text { reducing } \\
\text { sugars } \\
(\%)\end{array}$ & $\begin{array}{c}\text { Total } \\
\text { Sugars } \\
(\%)\end{array}$ & $\begin{array}{c}\text { Dry } \\
\text { matter } \\
\text { Content } \\
(\%)\end{array}$ & $\begin{array}{c}\text { Total } \\
\text { soluble } \\
\text { solids } \\
(\%)\end{array}$ \\
\hline $\mathbf{P}_{1} \times \mathbf{P}_{2}$ & $-0.47 * *$ & $-0.30 * *$ & -0.11 & 0.34 & $1.06^{* *}$ & $0.57 * *$ & $-0.41 * *$ & 0.15 & $0.36 *$ & 0.22 \\
\hline $\mathbf{P}_{1} \times \mathbf{P}_{3}$ & $0.83^{* *}$ & 0.05 & 0.20 & 0.13 & $-1.22 * *$ & 0.06 & $0.44 * *$ & $0.53 * *$ & 0.07 & -0.05 \\
\hline $\mathbf{P}_{1} \times \mathbf{P}_{4}$ & $-0.60 * *$ & 0.01 & $0.30 *$ & -0.15 & $-0.59 * *$ & -0.10 & $0.45^{* *}$ & $0.34^{*}$ & $1.44 * *$ & $0.52 * *$ \\
\hline $\mathbf{P}_{1} \times \mathbf{P}_{5}$ & $-0.69 * *$ & -0.07 & 0.19 & 0.39 & $0.97 * *$ & $0.30 * *$ & $0.39 * *$ & $0.68 * *$ & -0.05 & $0.52 * *$ \\
\hline $\mathbf{P}_{1} \times \mathbf{P}_{6}$ & 0.07 & 0.11 & 0.17 & 0.12 & 0.08 & $0.52 * *$ & $-0.43 * *$ & 0.12 & -0.20 & $1.15 * *$ \\
\hline $\mathbf{P}_{1} \times \mathbf{P}_{7}$ & $-0.63 * *$ & 0.12 & 0.07 & $0.92 * *$ & $1.28 * *$ & -0.16 & $-0.43 * *$ & $-0.60 * *$ & -0.05 & 0.29 \\
\hline $\mathbf{P}_{2} \times \mathbf{P}_{3}$ & $-1.17 * *$ & 0.05 & 0.20 & 0.01 & $-0.46 * *$ & 0.00 & $-0.28 * *$ & -0.25 & $1.01 * *$ & 0.22 \\
\hline $\mathbf{P}_{2} \times \mathbf{P}_{4}$ & -0.20 & 0.10 & $0.37 *$ & -0.10 & $1.10 * *$ & $0.70 * *$ & $-0.23 * *$ & $0.45^{* *}$ & 0.05 & -0.15 \\
\hline $\mathbf{P}_{2} \times \mathbf{P}_{5}$ & $0.49 * *$ & 0.03 & -0.04 & 0.40 & $-1.37 * *$ & $-0.83 * *$ & -0.03 & $-0.87 * *$ & $-0.37 *$ & $0.49 * *$ \\
\hline $\mathbf{P}_{2} \times \mathbf{P}_{6}$ & -0.19 & -0.09 & -0.03 & 0.16 & $2.30 * *$ & $0.22 *$ & $0.15^{*}$ & $0.40^{*}$ & -0.25 & $0.46 * *$ \\
\hline $\mathbf{P}_{2} \times \mathbf{P}_{7}$ & $-0.39 * *$ & $0.15^{* *}$ & 0.14 & $0.90 * *$ & $-0.54 * *$ & $-0.46 * *$ & $-0.28 * *$ & $-0.75 * *$ & $0.97 * *$ & $0.32 *$ \\
\hline $\mathbf{P}_{3} \times \mathbf{P}_{4}$ & 0.07 & 0.05 & 0.15 & 0.03 & $1.49 * *$ & $-0.38 * *$ & $-0.12 *$ & $-0.47 * *$ & 0.09 & -0.11 \\
\hline $\mathbf{P}_{3} \times \mathbf{P}_{5}$ & $-0.68 * *$ & 0.04 & 0.01 & $1.83 * *$ & $1.41 * *$ & 0.03 & 0.02 & 0.08 & $-0.33 *$ & $0.39 *$ \\
\hline $\mathbf{P}_{3} \times \mathbf{P}_{6}$ & $1.48 * *$ & -0.05 & 0.15 & -0.14 & 0.25 & -0.19 & $0.50 * *$ & 0.06 & $0.82 * *$ & 0.16 \\
\hline $\mathbf{P}_{3} \times \mathbf{P}_{7}$ & $-0.53 * *$ & -0.13 & 0.02 & $1.86^{* * *}$ & $-0.92 * *$ & -0.04 & 0.04 & 0.03 & -0.10 & $0.36^{*}$ \\
\hline $\mathbf{P}_{4} \times \mathbf{P}_{5}$ & $1.12 * *$ & 0.03 & $0.48 * *$ & $1.05 * *$ & $-0.92 * *$ & $0.19 *$ & $0.17 * *$ & $0.35^{*}$ & 0.18 & $0.62 * *$ \\
\hline $\mathbf{P}_{4} \times \mathbf{P}_{6}$ & $0.61 * *$ & -0.02 & -0.02 & $2.58 * *$ & 0.12 & $0.41 * *$ & 0.01 & $0.44 *$ & $0.86^{* *}$ & $0.59 * *$ \\
\hline $\mathbf{P}_{4} \times \mathbf{P}_{7}$ & -0.02 & 0.02 & 0.09 & 0.15 & $-0.69 * *$ & $-0.40 * *$ & $0.15^{*}$ & -0.25 & -0.29 & -0.11 \\
\hline $\mathbf{P}_{5} \times \mathbf{P}_{6}$ & $-0.77 * *$ & 0.10 & 0.21 & -0.55 & -0.02 & $0.45^{* *}$ & 0.12 & $0.58 * *$ & 0.31 & 0.19 \\
\hline $\mathbf{P}_{5} \times \mathbf{P}_{7}$ & -0.01 & -0.05 & 0.01 & $-1.12 * *$ & $1.40 * *$ & $0.47 * *$ & $-0.41 * *$ & 0.05 & $0.35^{*}$ & $0.49 * *$ \\
\hline $\mathbf{P}_{6} \times \mathbf{P}_{7}$ & $-1.38 * *$ & -0.07 & $0.49 * *$ & $-1.99 * *$ & 0.11 & $-0.28 * *$ & $-0.40 * *$ & $-0.66 * *$ & 0.07 & 0.26 \\
\hline $\operatorname{SE}\left(\mathbf{S}_{\mathrm{ij}}\right)$ & 0.11 & 0.10 & 0.14 & 0.31 & 0.16 & 0.09 & 0.06 & 0.16 & 0.16 & 0.14 \\
\hline $\operatorname{SE}\left(\mathbf{S}_{\mathrm{ij}} \mathbf{S}_{\mathrm{ik}}\right)$ & 0.17 & 0.15 & 0.20 & 0.45 & 0.23 & 0.14 & 0.09 & 0.23 & 0.23 & 0.21 \\
\hline
\end{tabular}

*, ** Significant at 5 per cent and 1 per cent probability levels, respectively

$\left(\mathrm{P}_{1}\right.$ - Narendra Agrim, $\mathrm{P}_{2}$ - Narendra Amrit, $\mathrm{P}_{3}$ - Narendra Upkar, $\mathrm{P}_{4}-$ NDPK-76-1, $\mathrm{P}_{5}-$ NDPK-724, $\mathrm{P}_{6}-$ NDPK-39-2, $\mathrm{P}_{7}$ - NDPK-12-10) 
Table.4 Estimates of components of variation and their related statistics in $7 \times 7$ diallel crosses of pumpkin

\begin{tabular}{|c|c|c|c|c|c|c|c|c|c|c|}
\hline $\begin{array}{l}\text { Components of variation and } \\
\text { related statistics }\end{array}$ & $\begin{array}{l}\text { Node no. to } 1^{\text {st }} \\
\text { male flower }\end{array}$ & $\begin{array}{l}\text { Node no. to } 1^{\text {st }} \\
\text { female flower }\end{array}$ & $\begin{array}{l}\text { Days to } 1^{\text {st }} \text { male } \\
\text { flower anthesis }\end{array}$ & $\begin{array}{l}\text { Days to } 1^{\text {st }} \\
\text { female flower } \\
\text { anthesis }\end{array}$ & $\begin{array}{l}\text { Days to } \\
\text { first fruit } \\
\text { harvest }\end{array}$ & $\begin{array}{c}\text { Number of } \\
\text { primary } \\
\text { branches per } \\
\text { plant }\end{array}$ & $\begin{array}{l}\text { Equatorial } \\
\text { circumferen } \\
\text { ce of fruit }\end{array}$ & $\begin{array}{c}\text { Polar } \\
\text { circumferen } \\
\text { ce of fruit }\end{array}$ & $\begin{array}{c}\text { Flesh } \\
\text { thickness }\end{array}$ & $\begin{array}{l}\text { Inter } \\
\text { nodal } \\
\text { length } \\
(\mathrm{cm})\end{array}$ \\
\hline$\hat{\mathrm{D}}_{\text {(Additive effect) }}$ & $2.08 *$ & $11.59 *$ & $21.44 *$ & $11.05^{*}$ & 8.33 & $1.12 *$ & $39.9 *$ & $28.27 *$ & $0.19 *$ & $0.76 *$ \\
\hline$\hat{H}_{1}$ (Dominance effect) & $2.66 *$ & $25.40 *$ & $39.65 *$ & $15.77 *$ & 22.14 & $2.39 *$ & $31.77 *$ & $29.24 *$ & $0.18 *$ & $1.63 *$ \\
\hline $\begin{array}{c}\hat{\mathrm{H}}_{2} \\
\text { (Dominance indicating } \\
\text { asymmetry of }+/- \text { effect of genes) }\end{array}$ & $1.78 *$ & $23.52 *$ & $31.46^{*}$ & $13.22 *$ & 20.48 & $2.13 *$ & $29.82 *$ & $27.03 *$ & $0.18^{*}$ & $1.51 *$ \\
\hline$\underset{\text { (Mean Fr over arrays) }}{\hat{\mathrm{F}}}$ & $-2.20 *$ & 6.15 & -8.10 & -1.52 & -0.21 & 0.69 & 10.18 & 10.44 & $-0.03 *$ & -0.02 \\
\hline$\hat{\mathrm{h}}^{2}$ & $2.17 *$ & 0.62 & 5.74 & -0.64 & 2.30 & $6.44 *$ & $39.47 *$ & $78.87 *$ & $0.66^{*}$ & 0.29 \\
\hline $\begin{array}{c}\hat{\mathrm{E}} \\
\text { (Environmental component) }\end{array}$ & 0.03 & 0.86 & 3.24 & $2.83^{*}$ & $5.30 *$ & 0.10 & $4.62 *$ & $3.61 *$ & $0.02 *$ & 0.11* \\
\hline $\begin{array}{c}\left(\hat{\mathrm{H}}_{1} / \hat{\mathrm{D}}\right)^{1 / 2} \\
\text { (Mean degree of dominance) }\end{array}$ & 1.28 & 2.19 & 1.85 & 1.43 & 1.63 & 1.46 & 0.89 & 1.01 & 0.97 & 1.46 \\
\hline $\begin{array}{c}\hat{\mathrm{H}}_{2} / 4 \hat{\mathrm{H}}_{1} \\
\begin{array}{c}\text { (Proportion of genes with }+/- \\
\text { effects in parents) }\end{array}\end{array}$ & 0.17 & 0.23 & 0.19 & 0.21 & 0.23 & 0.22 & 0.24 & 0.23 & 0.26 & 0.23 \\
\hline $\begin{array}{c}\left(4 \hat{\mathrm{D}} \hat{\mathrm{H}}_{1}\right)^{1 / 2}+\hat{\mathrm{F}}_{/\left(4 \mathrm{D} \hat{\mathrm{H}}_{1}\right)^{1 / 2}-} \hat{\mathrm{F}} \\
\begin{array}{c}\text { (Proportion of dominant and } \\
\text { recessive genes in parents) }\end{array}\end{array}$ & 0.76 & 0.89 & 0.36 & 1.44 & 0.99 & 1.53 & 1.33 & 1.44 & 0.85 & 0.98 \\
\hline $\begin{array}{c}\hat{\mathrm{h}}^{2} / \hat{\mathrm{H}}_{2} \\
\text { (Number of gene groups) }\end{array}$ & 0.18 & -0.05 & 1.22 & 0.03 & 0.11 & 3.01 & 1.32 & 2.92 & 3.56 & 0.19 \\
\hline $\mathbf{t}^{2}$ & 17.87 & 0.08 & 1.25 & 17.24 & 3.20 & 0.02 & 0.30 & 0.01 & 0.53 & 1.35 \\
\hline (1-b/SEb) & 2.27 & 2.46 & 2.09 & 2.36 & 5.98 & 3.27 & 3.15 & 1.47 & 2.42 & 2.59 \\
\hline
\end{tabular}

$*$,** Significant at 5 per cent and 1 per cent probability levels, respectively. 
Table.4 Contd...

\begin{tabular}{|c|c|c|c|c|c|c|c|c|c|c|}
\hline $\begin{array}{c}\text { Components of variation and } \\
\text { related statistics }\end{array}$ & $\begin{array}{l}\text { Vine } \\
\text { length } \\
\text { (m) }\end{array}$ & $\begin{array}{l}\text { Average } \\
\text { fruit } \\
\text { weight }\end{array}$ & $\begin{array}{l}\text { No. of } \\
\text { fruits } \\
\text { per } \\
\text { plant }\end{array}$ & $\begin{array}{l}\text { Fruit } \\
\text { yield per } \\
\text { plant }\end{array}$ & $\begin{array}{l}\text { Dry } \\
\text { matter } \\
\text { content }\end{array}$ & $\begin{array}{c}\text { Total } \\
\text { soluble } \\
\text { solids }\end{array}$ & $\begin{array}{c}\text { Total } \\
\text { Sugars }\end{array}$ & $\begin{array}{l}\text { Reducing } \\
\text { sugars }\end{array}$ & $\begin{array}{l}\text { Non- } \\
\text { reducing } \\
\text { sugars }\end{array}$ & Ascorbic acid \\
\hline$\stackrel{\hat{\mathrm{D}}}{\text { (Additive effect) }}$ & $1.13^{*}$ & $0.02 *$ & $0.22 *$ & $1.48 *$ & $0.96^{*}$ & 0.20 & 0.14 & 0.08 & $0.17^{*}$ & 0.30 \\
\hline $\begin{array}{c}\hat{\mathrm{H}}_{1} \\
\text { (Dominance effect) }\end{array}$ & $3.09 *$ & 0.01 & $0.27^{*}$ & $5.38 *$ & $1.67 *$ & $1.53 *$ & $1.56^{*}$ & $0.91 *$ & $0.66^{*}$ & $4.95 *$ \\
\hline $\begin{array}{c}\hat{\mathrm{H}}_{2} \\
\text { (Dominance indicating asymmetry } \\
\text { of }+/ \text { - effect of genes) }\end{array}$ & $2.36^{*}$ & 0.02 & $0.26^{*}$ & $4.74 *$ & $1.48 *$ & $1.32 *$ & $0.94 *$ & $0.67 *$ & $0.43^{*}$ & $4.65^{*}$ \\
\hline$\hat{\mathrm{F}}$ (Mean Fr over arrays) $\backslash$ & $1.27 *$ & -0.01 & -0.06 & 0.89 & 0.70 & 0.33 & 0.39 & 0.17 & 0.26 & 0.54 \\
\hline$\hat{\mathrm{h}}^{2}$ & $0.99 *$ & -0.005 & $0.26^{*}$ & $4.91 *$ & $2.61 *$ & $4.94 *$ & 0.002 & 0.12 & 0.04 & 2.49 \\
\hline $\begin{array}{c}\hat{\mathrm{E}} \\
\text { (Environmental component) }\end{array}$ & 0.02 & $0.01 *$ & $0.02 *$ & 0.12 & 0.03 & 0.03 & 0.03 & 0.01 & 0.004 & 0.03 \\
\hline $\begin{array}{c}\left(\hat{\mathrm{H}}_{1} / \hat{\mathrm{D}}\right)^{1 / 2} \\
\text { (Mean degree of dominance) }\end{array}$ & 1.65 & 0.67 & 1.09 & 1.90 & 1.32 & 2.73 & 3.28 & 3.33 & 1.99 & 4.03 \\
\hline $\begin{array}{c}\hat{\mathrm{H}}_{2} / 4 \hat{\mathrm{H}}_{1} \\
\text { (Proportion of genes with +/- effects } \\
\text { in parents) }\end{array}$ & 0.19 & 0.38 & 0.24 & 0.22 & 0.22 & 0.22 & 0.15 & 0.18 & 0.16 & 0.24 \\
\hline $\begin{array}{c}\left(4 \hat{\mathrm{D}} \hat{\mathrm{H}}_{1}\right)^{1 / 2}+\hat{\mathrm{F}} /\left(4 \hat{\mathrm{D}} \hat{\mathrm{H}}_{1}\right)^{1 / 2}-\hat{\mathrm{F}} \\
\begin{array}{c}\text { Proportion of dominant and } \\
\text { recessive genes in parents) }\end{array}\end{array}$ & 2.03 & 0.63 & 0.79 & 1.37 & 1.76 & 1.82 & 2.39 & 1.89 & 2.28 & 1.56 \\
\hline $\begin{array}{c}\hat{\mathrm{h}}^{2} / \hat{\mathrm{H}}_{2} \\
\text { (Number of gene groups) }\end{array}$ & 0.42 & -0.34 & 3.83 & 1.04 & 1.77 & 3.76 & 0.003 & 0.18 & 0.09 & 0.54 \\
\hline $\mathbf{t}^{2}$ & 1.51 & 3.80 & 4.82 & 0.10 & 0.65 & 3.34 & 0.03 & 1.98 & 0.37 & 0.38 \\
\hline (1-b/SEb) & 3.08 & 0.65 & 1.51 & 1.56 & 3.49 & 2.99 & 2.32 & 7.47 & 2.14 & 4.48 \\
\hline
\end{tabular}

*, ** Significant at 5 per cent and 1 per cent probability levels, respectively. 
Perusal of data presented in Table- 3 revealed that out of $21 \mathrm{~F}_{1}$ hybrids, significant sca effects in favourable direction were exhibited by eleven hybrids for days to first male flower anthesis, for days to first female flower anthesis, node number to first male flower appearance, five crosses for node number to first female flower appearance, two hybrids for days to first fruit harvest, six hybrids for number of primary branches per plant, two hybrids for equatorial circumference of fruit, three hybrids for polar circumference of fruit, three hybrids for flesh thickness, two hybrids for internodal length, five hybrids for vine length, one for average fruit weight, four hybrids hybrids for number of fruits per plant, seven for dry matter content, eleven hybrids for total soluble solids, eight hybrids for total sugars, one for reducing sugars, seven hybrids for non-reducing sugars, eight hybrid crosses for ascorbic acid. Significant SCA effects had also been reported for these traits by earlier scientists.

The SCA effect of the cross is an estimate value while per se performance is the realized value, the later should also be given due to consideration, while making selection of best cross combinations. The best five crosses on the basis of desirable and significant SCA effects and per se performance with general combining ability effects for all the traits has been presented in Table-3. This table revealed that the crosses with the best per se performance did not necessarily have good SCA effect i.e. the best specific combiner might or might not give the best per se performance in the crosses. For instance the best specific combinations $\mathrm{P}_{1} \times \mathrm{P}_{4}, \mathrm{P}_{1} \times \mathrm{P}_{5}, \mathrm{P}_{1}$ $\times \mathrm{P}_{6}$, and $\mathrm{P}_{2} \times \mathrm{P}_{7}$ for days to first male flower anthesis, $\mathrm{P}_{1} \times \mathrm{P}_{6}, \mathrm{P}_{5} \times \mathrm{P}_{7}$ for days to first female flower anthesis, $\mathrm{P}_{1} \times \mathrm{P}_{4}, \mathrm{P}_{1} \times \mathrm{P}_{6}, \mathrm{P}_{2} \mathrm{x}$ $\mathrm{P}_{4}, \mathrm{P}_{3} \times \mathrm{P}_{5}, \mathrm{P}_{5} \times \mathrm{P}_{6}$ for node number to first male flower appearance, $\mathrm{P}_{2} \times \mathrm{P}_{7}, \mathrm{P}_{3} \times \mathrm{P}_{5}, \mathrm{P}_{3} \times$ $\mathrm{P}_{7}, \mathrm{P}_{5} \times \mathrm{P}_{6}, \mathrm{P}_{5} \times \mathrm{P}_{7}$ for node number to first female flower appearance, $\mathrm{P}_{1} \times \mathrm{P}_{4}, \mathrm{P}_{1} \times \mathrm{P}_{6}$ for days to first fruit harvest, $\mathrm{P}_{1} \times \mathrm{P}_{3}, \mathrm{P}_{1} \times \mathrm{P}_{6}, \mathrm{P}_{2} \times$ $\mathrm{P}_{4}, \mathrm{P}_{3} \times \mathrm{P}_{6}, \mathrm{P}_{3} \times \mathrm{P}_{7}, \mathrm{P}_{4} \times \mathrm{P}_{5}$ for primary branches per plant, $\mathrm{P}_{1} \times \mathrm{P}_{3}, \mathrm{P}_{2} \times \mathrm{P}_{5}$ for equatorial circumference of fruit, $\mathrm{P}_{1} \times \mathrm{P}_{6}, \mathrm{P}_{4} \times$ $\mathrm{P}_{5}, \mathrm{P}_{6} \times \mathrm{P}_{7}$ for polar circumference of fruit, $\mathrm{P}_{1}$ $\times \mathrm{P}_{2}, \mathrm{P}_{3} \times \mathrm{P}_{7}, \mathrm{P}_{4} \times \mathrm{P}_{6}$ for flesh thickness, $\mathrm{P}_{1} \times$ $\mathrm{P}_{3}, \mathrm{P}_{2} \times \mathrm{P}_{5}$ for internodal length, $\mathrm{P}_{1} \times \mathrm{P}_{3}, \mathrm{P}_{2} \times$ $\mathrm{P}_{5}, \mathrm{P}_{3} \times \mathrm{P}_{6}, \mathrm{P}_{4} \times \mathrm{P}_{5}, \mathrm{P}_{4} \times \mathrm{P}_{6}$ for vine length, $\mathrm{P}_{2}$ $x \mathrm{P}_{7}$ for average fruit weight, $\mathrm{P}_{1} \times \mathrm{P}_{4}, \mathrm{P}_{2} \times \mathrm{P}_{4}$, $\mathrm{P}_{4} \times \mathrm{P}_{5}, \mathrm{P}_{6} \times \mathrm{P}_{7}$ for number of fruits per plant, $\mathrm{P}_{1} \times \mathrm{P}_{7}, \mathrm{P}_{2} \times \mathrm{P}_{7}, \mathrm{P}_{3} \times \mathrm{P}_{5}, \mathrm{P}_{3} \times \mathrm{P}_{7}, \mathrm{P}_{4} \times \mathrm{P}_{5}, \mathrm{P}_{4} \times$ $\mathrm{P}_{7}$, for fruit yield per plant, $\mathrm{P}_{1} \times \mathrm{P}_{2}, \mathrm{P}_{1} \times \mathrm{P}_{4}, \mathrm{P}_{2}$ $\times \mathrm{P}_{3}, \mathrm{P}_{2} \times \mathrm{P}_{7}, \mathrm{P}_{2} \times \mathrm{P}_{7,}, \mathrm{P}_{3} \times \mathrm{P}_{6}, \mathrm{P}_{4} \times \mathrm{P}_{6}, \mathrm{P}_{5} \times \mathrm{P}_{7}$ for dry matter content, $\mathrm{P}_{1} \times \mathrm{P}_{4}, \mathrm{P}_{1} \times \mathrm{P}_{5}, \mathrm{P}_{1} \times$ $\mathrm{P}_{6}, \mathrm{P}_{2} \times \mathrm{P}_{5}, \mathrm{P}_{2} \times \mathrm{P}_{6}, \mathrm{P}_{2} \times \mathrm{P}_{7}, \mathrm{P}_{3} \times \mathrm{P}_{5}, \mathrm{P}_{3} \times \mathrm{P}_{7}$, $\mathrm{P}_{4} \times \mathrm{P}_{5}, \mathrm{P}_{4} \times \mathrm{P}_{6}, \mathrm{P}_{5} \times \mathrm{P}_{7}$ for total soluble solids, $\mathrm{P}_{1} \times \mathrm{P}_{3}, \mathrm{P}_{1} \times \mathrm{P}_{4}, \mathrm{P}_{1} \times \mathrm{P}_{5}, \mathrm{P}_{2} \times \mathrm{P}_{4}, \mathrm{P}_{2} \times$ $\mathrm{P}_{6}, \mathrm{P}_{4} \times \mathrm{P}_{5}, \mathrm{P}_{4} \times \mathrm{P}_{6}, \mathrm{P}_{5} \times \mathrm{P}_{6}$ for total sugars, $\mathrm{P}_{2}$ $\mathrm{x} \mathrm{P}_{4}$ for reducing sugars, $\mathrm{P}_{1} \times \mathrm{P}_{3}, \mathrm{P}_{1} \times \mathrm{P}_{4}, \mathrm{P}_{1} \times$ $\mathrm{P}_{5}, \mathrm{P}_{2} \times \mathrm{P}_{6}, \mathrm{P}_{3} \times \mathrm{P}_{6}, \mathrm{P}_{4} \times \mathrm{P}_{5}, \mathrm{P}_{4} \times \mathrm{P}_{7}$ for nonreducing sugars and $\mathrm{P}_{1} \times \mathrm{P}_{2}, \mathrm{P}_{1} \times \mathrm{P}_{5}, \mathrm{P}_{1} \times \mathrm{P}_{7}$, $\mathrm{P}_{2} \times \mathrm{P}_{4}, \mathrm{P}_{2} \times \mathrm{P}_{6}, \mathrm{P}_{3} \times \mathrm{P}_{4}, \mathrm{P}_{3} \times \mathrm{P}_{5}, \mathrm{P}_{5} \times \mathrm{P}_{7}$ for ascorbic acid. The significant and desirable SCA effects have been also reported by earlier researchers for various triats viz., fruit yield per plant (Maurya, 2006; Nisha and Veeraragavathatham, 2014) maturity traits (Nisha and Veeraragavathatham, 2014) fruit weight (Dubey and Maurya, 2006) flesh thickness (Sreevani, 2005) which supported the present investigation.

The estimates of the components of variation and their related statistics for different traits of pumpkin have been presented in Table- 4 .

Highly significant values for additive ( $\hat{D})$ and dominance ( $\hat{\mathbf{H}}_{1}$ and $\left.\hat{\mathbf{H}}_{2}\right)$ effects of components were observed for most of the twenty traits except the values of $\hat{D}$ for days to first fruit harvest, total soluble solids, total sugar and ascorbic acid in which these parameters were found non-significant. The significant values of $\hat{D}, \hat{\mathbf{H}}_{1}$ and $\hat{\mathbf{H}}_{2}$ indicated the importance of both additive and 
dominance gene action in the expression of these traits, which is in consonance with the findings of Kathiria et al., (2005) and Jha et al., (2009). However, additive ( $\hat{\mathrm{D}})$ genetic variance components were lower in magnitude than dominant component of genetic variance for all the twenty traits except flesh thickness, average fruit weight which showed preponderance of dominance components of variance in expression of fruit yield and its attributing traits. Similar findings were also recorded by Sharma et al., (2010) in pumpkin.

The positive values of $\hat{F}$ were found for all traits except days to male flower anthesis, days to first female flower anthesis, node number to first male flower anthesis, days to first fruit harvest, flesh thickness, intermodal length, average fruit weight and number of fruits per plant which indicated that there were an excess of dominance gene in the inheritance of these traits among the parents. The average degree of dominance $\left(\hat{H}_{1 / \hat{D}}\right)^{1 / 2}$ revealed the presence of over dominance for all characters. This suggested that heterosis breeding might be advantageous for improvement of yield and its attributing traits in pumpkin. The results are in agreement with the finding of Jha et al., (2009) and Sharma et al., (2010).

Ratio of $\left(\hat{\mathrm{H}}_{2 / 4} \hat{\mathrm{H}}_{1}\right)$ which estimates frequency of alleles with positive and negative effects in the parents were less than 0.25 for all characters which showed asymmetrical distribution of loci showing dominance for all traits (Table-4). The ratio of $\left(4 \hat{\mathrm{D}}^{\hat{\mathrm{H}}_{1}}\right)^{1 / 2}+\hat{\mathrm{F}} /$ $\left(4 \hat{D}^{\hat{H}_{1}}\right)^{1 / 2}{ }_{-} \hat{F}$ indicated that the dominant alleles were more frequent than recessive alleles for all the traits studied (Table-4). These findings are similar to that of Sharma et al., (2010).
The proportion of $\hat{\mathrm{h}}^{2} / \hat{\mathrm{H}}_{2}$, which provides information about groups of gene exhibiting little or no dominance. The less than one $\hat{\mathrm{h}}^{2} / \hat{\mathrm{H}}_{2}$ ratio suggested that atleast one gene group mainly governed the characters under study for most of traits. The positive correlation ( $r$ ) between parental order of dominance $(\mathrm{Wr}+\mathrm{Vr})$ and parental measurement (Yr) were showed for most of the characters, except days to first female flower anthesis and average fruit weight. The positve correlation suggesting the preponderance of recessive genes while, positive values suggested preponderance of dominant genes. The results of present investigation suggested preponderance of dominant genes in the expression of most of traits studied. Therefore, heterosis breeding approach could be advantageous to produce superior hybrids for high fruit yield in pumpkin. The above findings are in agreement with that of Singh et al., (2005).

Thus the non-significant values of ' $\mathrm{t}$ ', or (1$\mathrm{b} / \mathrm{SEb}$ ) indicates the validity of assumptions pertaining to diallel analysis, while significant values of ' $\mathrm{t}$ ', or (1-b/SEb) showed failure of hypothesis or null hypothesis for diallel cross analysis (Table-4).

The result of present study suggested preponderance of dominance genes in the expression of most of the component traits studied. Therefore, heterosis breeding approach might be advantageous rather than selection to develop superior hybrids for high fruit yield in pumpkin.

\section{Acknowledgment}

The work on pumpkin reported in this paper has been supported by research and teaching faculties of Department of Vegetable Science, N.D.U.A.T and We would also like to thank Mr. Murli Mohan Khetan for statistical analysis. 


\section{References}

Adeoye A.A. and Coyne D.P. (1981) Inheritance of resistance to trifluralin toxicity in colour in Cucurbita moschata. Horticultura Brasileira 13, 170-171.

Anonymous. (2015). Indian Horticulture Database. National Horticulture Board, Ministry of Agriculture \& farmer welfare, Govt. of India, Gurgaon.

Arunachalam, V. (1989). Genetic basis of plant breeding. In Plant Breeding Theory and Practice (ed.) V. L. Chopra, Oxford and IBH Publishing Co. Ltd., New Delhi.

Cardoso AII, Silva N, Della-Vecchia PT (1995) Inheritance of immature fruit characters in pumpkin (C. moschata) Vegetable Science 14: 143-146.

Doijode S.D, Sulladmath U.V (1988) Genetics of certain vegetative and flowering characters in pumpkin $(C$. moschata). Agricultural Science Digest 8, 203-206.

Doijode SD, Sulladmath UV, Kulkarnio RS (1987). Inheritance of certain seed characters in pumpkin (C. moschata) Vegetable Science 14: 143-146.

Dubey, S.K. and Maurya, I.B. (2006). Studies on combining ability in bottle gourd. Orissa J. Hort., 34 (1): 81-85.

Ferriol and Pico (2008). 'Pumpkin and Winter Squash' A Book Chapter in Vegetable I pp. 317-349. Edited by Jaime Prohens and Fernando Nuez Springer Science + Business Media.

Galka Al (1987). Inheritance of some economically useful traits in first generation hybrids of Cucurbita. Oveshchevedstvo I Bakhchevodstvo 32, 45-46.

Jha, A., Pandey, S., Rai M., Yadav, D.S. and Singh, T.B. (2009). Combining ability for flowering behaviors and yield parameters in pumpkin. Vegetable
Science, 36: 332-335.

Kathiria, K.B., Acharya, R.R., Patel, A.D., Nag, M. and Bhalala, M.K. (2005) Genetic analysis in bottle gourd. In: Abstract Book of National Seminar on Cucurbits, Sept. 22-23, G.B. Pant University of Agriculture and Technology, Pantnagar. p. 10.

Kumar, R. (2000). Effect of seasonal variation on the performance of various inbreds and their hybrids of bottle gourd. Ph.D. Thesis, Submitted to N.D. Univ. of Agric. \& Tech., Kumarganj, Faizabad.

Latha P and Gopala krishnan TR (1993) Inheritance of mosaic diseases and silvery leaf trait in pumpkin (Cucurbita moschata Poir). Horticultural Journal 6: 97-101.

Maurya, I.B. (1994). Combining ability analysis in bottle gourd (Lagenaria siceraria (Molina.) standl.). Ph.D. Thesis, Submitted to N.D. Univ. of Agric. \& Tech., Kumarganj, Faizabad.

Maurya, I.B. (2006). Studies on heterosis and combining ability in bottle gourd. M.Sc. Thesis, submitted to N.D. Univ. of Agric \& Tech., Kumarganj, Faizabad.

Nisha, S.K. and Veeraragavathatham, D. (2014). Heterosis and combining ability for fruit yield and its component traits inpumpkin (Cucurbita moschata Duch. ex Poir.) Advances in Applied. Research. 6 (2): 158 - 162.

Pandey, S.K., Srivastava, A.K., Tiwari, J.K. and Srivastava, J.P. (2010). Studies on heterosis and combining ability for earliness in Bottle gourd [Lagenaria siceraria (Molina) Standl.]. International Seminar on Rec. Trend Hi-Tech. Hort. \& PHT, Kanpur, p. 45.

Panse, V.G. and Sukhatme, P.V. (1967). Statistical Methods for Agriculture Workers. Indian Council of Agriculture Research, New Delhi.

Paris HS, Brown RN (2005). The genes of pumpkin and squash. HortScience 40: 
1620-1630.

Rehana, H. and Sharma, S.P. (2007). Combining ability studies in bottle gourd. Crop Res. 33: 148-151.

Ribeiro A, da Costa CP (1989) Inheritance of mottled leaf in Cucurbita moschata. Report of the Cucurbit Genetics Cooperative 12, 70-71.

Sharma, N., Sharma, N.K. and Malik, Y.S. (2010). Estimation of genetic variation in bottle gourd, In : Abstract book of National Seminar on Recent Trends in Hort. Crops Issues and Strategies for Res. Development, March 22-24, CCS HAU, Hissar, p. 26.

Singh, V.B., Mishra, C.H., Singh, S.P. and Srivastava, R.K. (2005). Combining ability in bottle gourd (Lagenaria siceraria (Mol.) Standl.). In: Abstract Book of National Seminar on Cucurbits, Sept. 22-23, G.B. Pant University of Agriculture and Technology, Pantnagar. p.162.
Sirohi P.S, Kumar T.S. and Choudhury B. (1986) Diallel analysis for variability in Cucurbita moschata (Duch) Poir. Indian Journal of Agril. Sci. 56: 171173.

Sirohi P.S., Yayasani S.R. (2001) Gene action of mineral elements and vitamins in pumpkin (Cucurbita moschata). Vegetable Science 28: 127-129.

Sreevani, P.G. (2005). Combining ability analysis for yield and its components in bottle gourd (Lagenaria siceraria (Mol.) Standl.). Veg. Sci., 32 (2): 140142.

Wessel-Beaver L and Katzir N (2000) Inheritance of silverleaf resistance in Cucurbita moschata. Acta Horticulturae 510: 289-295.

Zhou, S.K., Qiu, Z.H., Li, G.X., Li, Z. and Li, X.R. (1995). Study on the germplasm resources of Cucurbita for seed and its utilization. Crop. Genet. Res., 2: 13.

\section{How to cite this article:}

Singh, M.K., V.B. Singh, G.C. Yadav and Pushpendra Kumar. 2019. To Study the Combining Ability and Gene Action Involve in Expression of Traits in Pumpkin (Cucurbita moschata Duch. ex. Poir). Int.J.Curr.Microbiol.App.Sci. 8(06): 1535-1549. doi: https://doi.org/10.20546/ijcmas.2019.806.185 